3-5 novembre 1992, Lyon (France)

Novatech 92: Nouvelles technologies en assainissement pluvial ( $1^{\text {ere }}$ conf. européenne)

Renseignements: G.R.A.I.E., B.P. 2132 69603 Villeurbanne Cedex, France.

3-6 novembre 1992, Lyon (France) Pollutec $92\left(3^{\mathrm{e}}\right.$ salon international des techniques : eau, air, bruit, déchets, nettoyage industriel).

Organisation: Technoexpo, 8 rue de la Michodière, 75002 Paris, France. Tél. : (1) 47429256 - Fax : 42661428

3-6 novembre 1992, Séville (Espagne) BEM 14 (14th Boundary elements int. conf.)

Renseignements: Sue Owen, Conference Secretariat, Wessex Institute of Technology, Ashurst Lodge, Ashurst, Southampton, SO4 2AA, UK. Tél. : (0703) 293223 - International Tél. : 44703293223 - Fax : (0703) 292853 - International Fax : 44703292853

3-15 novembre 1992, Erice, Sicile (Italie) Coping with floods

Renseignements: Prof. Giuseppe Rossi, Istituto di Idraulica, Idrologia e Gestione delle Acque, Università di Catania, Viale Andrea Doria 6, 95125 Catania, Italie. Tél. : (39) 095256424 - Fax: (39) 95339225 .

8-13 novembre 1992, Anaheim, Ca. (USA) Flow induced vibrations and noise (int. symp.)

Renseignements : Prof. Michael P. Paĩdoussis, Dept. of Mechanical Engineering, McGill University, 817 Sherbrooke Street West, Montreal, QC, Canada H3A 2K6. Tél. : (1) 514 398-6294 - Fax : (1) 514398 7365 .

10-12 novembre 1992, Vienne (Autriche) Water energy plants ( 7 th int. sem.)

Renseignements : Institut für Wasserkraftmaschinen und Pumpen, Technische Universität Wien, z. Hd. Dr. Ch. Fuhrmann, Karlsplatz 13/305, A-1040 Vienne, Autriche. Tél. : +4322258801 3295 - Fax : +432225041148 .

17-20 novembre 1992, Wuhan (Chine) Hydraulic research in nature and laboratory (int. symp.)

Thèmes: River dynamics. - Hydraulics for structures and hydromachines. - Environmental hydraulics. - Cooling water system. - Navigation hydraulics. Groundwater hydraulics.

Renseignements : Prof. Liu Daming, Yangtze River Scientific Research Institute, 23 Huang Pu Road, Wuhan 430010, Chine. Télex : 40139.

9-11 décembre 1992, Cambridge (UK) Cavitation (3rd int. conf.)

Renseignements: Hazel Anderson/Anne Nolan, Conference Department C453, Institution of Mechanical Engineers, $1 \mathrm{Bir}$ dcage Walk, Londres SW1H 9JJ. Tél. : 071$9731317 / 1249$, Fax : 071-222 9881.

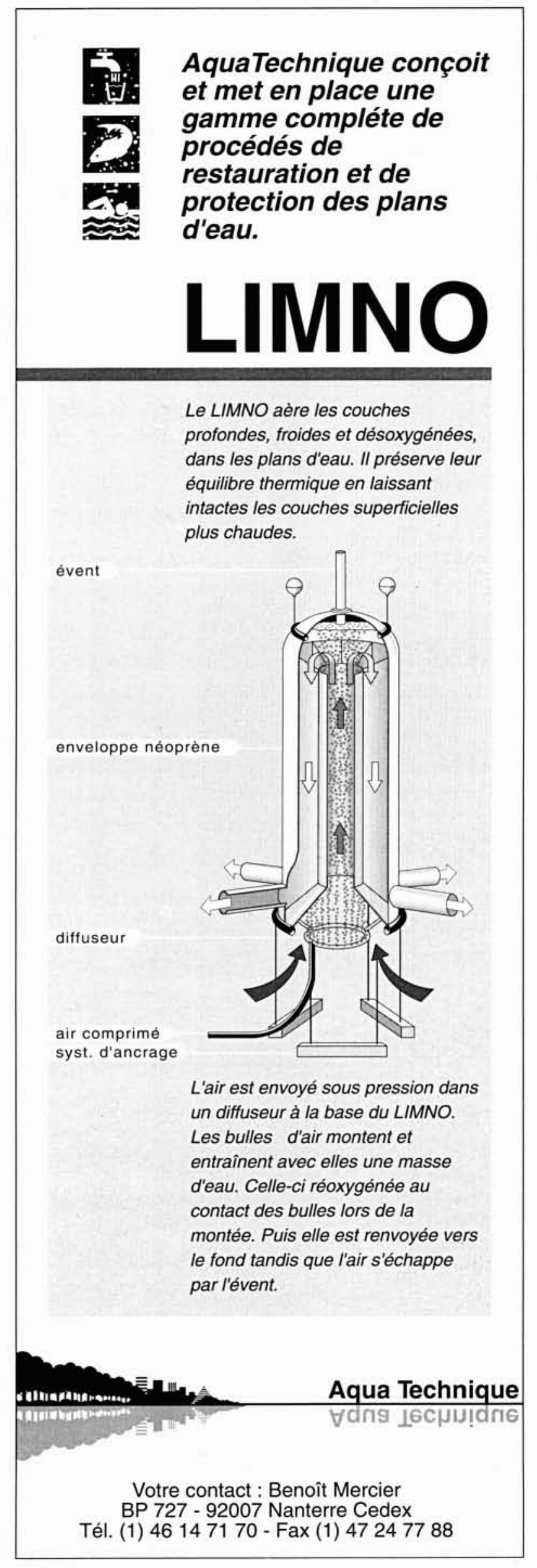

LA HOUILLE BLANCHE/N $1-1992$ 


\title{
Les grandes machines tournantes hydrauliques
}

\begin{abstract}
A u cours des 20 dernières années, les constructeurs de turbines hydrauliques ont été conduits à mettre au point des machines tournantes particulièrement grandes. Cette évolution a concerné la plupart des types de machines et pas seulement les turbines de très fortes puissances, de type Francis.

L'équipement des chutes de moins de $60 \mathrm{~m}$ a en effet poussé au développement des Kaplan, des Hélices et des Bulbes, dont les caractéristiques dimensionnelles sont parfois encore plus importantes que pour les Francis.

D'autre part, les besoins croissants d'adaptation de l'offre à la demande en matière d'électricité ont donné beaucoup d'intérêt aux stations de stockage d'énergie équipées de turbines-pompes de grandes dimensions.

Il n'y a que les Pelton qui n'ont pas été entraînées dans la course au gigantisme. Du fait qu'elles fonctionnent sous haute ou très haute chute, leurs dimensions restent dans des limites classiques (diamètres de roues de 3500 à $4500 \mathrm{~mm}$ ).

Les tableaux ci-après, établis, en bonne partie, d'après Water Power and Dam Construction Handbook 1991, rassemblent quelques données sur des machines en service ou en construction ayant des caractéristiques exceptionnelles, qu'il s'agisse de puissance unitaire, de diamètre de roue ou de hauteur de chute.
\end{abstract}

\begin{tabular}{|c|c|c|c|c|c|}
\hline \multicolumn{6}{|l|}{ Francis } \\
\hline \multicolumn{6}{|c|}{$\begin{array}{l}\text { Puissance unitaire }>700 \mathrm{MW} \\
\text { ou Diamètre de roue }>8000 \mathrm{~mm} \\
\text { ou Hauteur de chute }>700 \mathrm{~m}\end{array}$} \\
\hline & & $\begin{array}{l}\text { Puissance } \\
\text { unitaire } \\
\text { MW }\end{array}$ & $\begin{array}{c}\varnothing \\
\text { roue } \\
\mathrm{mm}\end{array}$ & $\begin{array}{l}\text { Hauteur } \\
\text { de chute } \\
\mathrm{m}\end{array}$ & $\begin{array}{l}\text { Année } \\
1^{\text {re }} \text { mise en } \\
\text { service }\end{array}$ \\
\hline $\begin{array}{l}\text { Grand Coulée } 22.24 \\
\text { Itaipu } \\
\text { Guri } \\
\text { Tucurui } \\
\text { Hausling }\end{array}$ & $\begin{array}{l}\text { USA } \\
\text { Brésil-Paraguay } \\
\text { Vénézuéla } \\
\text { Brésil } \\
\text { Autriche }\end{array}$ & $\begin{array}{l}838 \\
740 \\
725 \\
369 \\
180\end{array}$ & $\begin{array}{l}\mathbf{9 9 0 0} \\
\mathbf{8 1 0 0} \\
6900 \\
\mathbf{8 1 5 0} \\
1800\end{array}$ & $\begin{array}{r}87 \\
118 \\
146 \\
68 \\
734\end{array}$ & $\begin{array}{l}1978 \\
1984 \\
1984 \\
1984 \\
1986\end{array}$ \\
\hline
\end{tabular}

La technique française est bien présente pour ces Francis exceptionnelles puisque :

- pour Tucurui (12 machines) la technique des turbines est $100 \%$ Neyrpic;

- pour Itaipu (18 machines) la technique des turbines est commune à Neyrpic et à Voith.

Pour les prochaines années, les projets de Francis en préparation (si l'on excepte celui des Trois Gorges en Chine) portent sur des machines de caractéristiques moins exceptionnelles avec, pour les plus grandes, des puissances comprises entre 250 et $600 \mathrm{MW}$. 


\begin{tabular}{|c|c|c|c|c|c|}
\hline \multicolumn{6}{|l|}{ Kaplan } \\
\hline \multicolumn{6}{|c|}{$\begin{array}{rr}\text { Puissance unitaire }> & 230 \mathrm{MW} \\
\text { ou Diametre de roue } & >10300 \mathrm{~mm}\end{array}$} \\
\hline & & $\begin{array}{c}\text { Puissance } \\
\text { unitaire } \\
\text { MW }\end{array}$ & $\begin{array}{c}\varnothing \\
\text { roue } \\
\mathrm{mm}\end{array}$ & $\begin{array}{c}\text { Hauteur } \\
\text { de chute } \\
\text { m }\end{array}$ & $\begin{array}{c}\text { Année } \\
1^{\mathrm{re}} \text { mise en } \\
\text { service }\end{array}$ \\
\hline $\begin{array}{l}\text { Shulbinskaya } \\
\text { Saratovskaya } \\
\text { Gezhouba }\end{array}$ & $\begin{array}{l}\text { URSS } \\
\text { URSS } \\
\text { Chine }\end{array}$ & $\begin{array}{r}230 \\
60 \\
170\end{array}$ & $\begin{array}{r}8500 \\
10300 \\
11300\end{array}$ & $\begin{array}{l}48 \\
15 \\
19\end{array}$ & 1983 \\
\hline
\end{tabular}

Du fait du manque de référence importante en France, Neyrpic a été longtemps absente des grandes réalisations de Kaplan et d'Hélices. Ce retard est maintenant comblé avec 22 Kaplan de plus de $100 \mathrm{MW}$ dont, en association avec Mecanica Pesada, les 18 Kaplan de Porto Primavera au Brésil. Une référence voisine mérite aussi d'être mentionnée : celle, toute récente, des 4 hélices de $114 \mathrm{MW}$ pour le contrat L.G.1 (Québec) dont Neyrpic assure l'hydraulique pour le compte de GEC Alsthom Electromécanique Inc (ex Marine Industrie Limitée).

Pour le proche futur, les études portent sur des machines dont les plus importantes ont des caractéristiques qui sont plutôt moins extrêmes que celles indiquées dans le tableau ci-dessus (puissances comprises entre 150 et $170 \mathrm{MW}$ pour des chutes en général supérieures à $30 \mathrm{~m}$ ).

\begin{tabular}{|c|c|c|c|c|c|}
\hline \multicolumn{6}{|l|}{ Bulbes } \\
\hline \multicolumn{6}{|c|}{$\begin{array}{cr}\text { Puissance unitaire } & > \\
\text { ou Diamètre de roue } & >8000 \mathrm{MW}\end{array}$} \\
\hline & & $\begin{array}{c}\text { Puissance } \\
\text { unitaire } \\
\text { MW }\end{array}$ & $\begin{array}{c}\varnothing \\
\text { roue } \\
\mathrm{mm}\end{array}$ & $\begin{array}{c}\text { Hauteur } \\
\text { de chute } \\
\text { m }\end{array}$ & $\begin{array}{c}\text { Année } \\
1^{\text {re }} \text { mise en } \\
\text { service }\end{array}$ \\
\hline $\begin{array}{l}\text { Tadami } \\
\text { Rock Island } \\
\text { Vidalia }\end{array}$ & $\begin{array}{l}\text { Japon } \\
\text { USA } \\
\text { USA }\end{array}$ & $\begin{array}{l}\mathbf{6 5} \\
\mathbf{5 3} \\
24\end{array}$ & $\begin{array}{l}6450 \\
7400 \\
\mathbf{8 2 0 0}\end{array}$ & $\begin{array}{r}21 \\
12 \\
8\end{array}$ & $\begin{array}{l}1989 \\
1974 \\
1990\end{array}$ \\
\hline
\end{tabular}

Malgré leurs faibles puissances unitaires, les bulbes peuvent, comme on le voit, conduire à des machines de très grands diamètres.

Les références acquises en France, grâce à EDF et à la CNR, sur le Rhône, le Rhin et la Rance, ont permis à Neyrpic d'être l'incontestable leader dans ce domaine des basses chutes avec $50 \%$ des machines de plus de $5 \mathrm{~m}$ de diamètre sur technique française dont les 8 turbines de Rock Island aux Etats-Unis.

Deux évolutions concernant les bulbes nécessitent d'être évoquées :

- l'équipement de très basses chutes jusqu'à 3,5 mètres. C'est maintenant possible avec des turbines-puits ayant des multiplicateurs pour entraîner des alternateurs rapides ;

- l'équipement de chutes de 20 à $30 \mathrm{~m}$ avec des bulbes de très fortes puissances unitaires, plus de $100 \mathrm{MW}$. Cette évolution qui ferait empiéter les bulbes sur le domaine traditionnel des Kaplan et des Hélices n'en est encore qu'au stade des prétudes. 


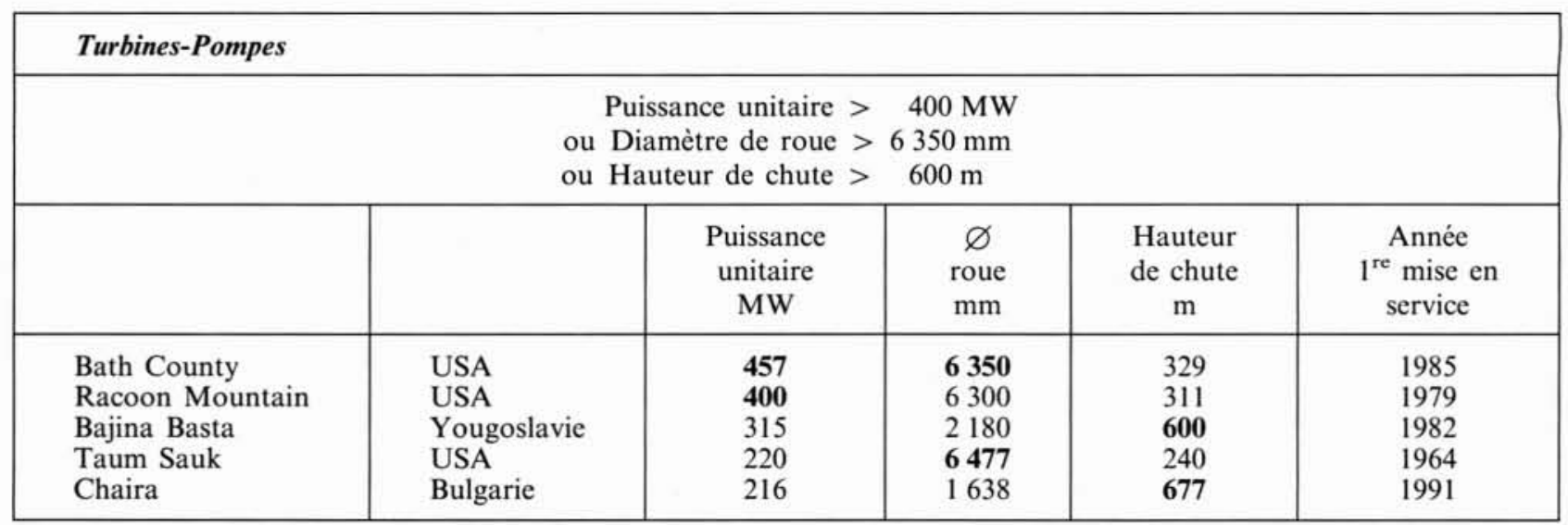

Il ne s'agit là que des turbines-pompes mono étage. Les caractéristiques dimensionnelles des turbines-pompes multi étages qui opèrent sous des hauteurs de chutes beaucoup plus fortes (1 $200 \mathrm{~m}$ en 5 étages à Super Bissorte) sont en effet très nettement plus réduites.

Comme pour les Kaplan, Neyrpic n'a réussi à percer dans le marché des turbines-pompes que tardivement à l'occasion du programme important lancé par EDF en France pour accompagner le développement des centrales nucléaires. Là aussi le retard est maintenant rattrapé avec notamment les belles commandes de Guangzhou en Chine $(4 \times 306 \mathrm{MW})$ et de Muju en Corée du Sud $(2 \times 330 \mathrm{MW})$.

Les projets de station de pompage pour accumulation d'énergie sont relativement importants pour les prochaines années. Ils portent sur des machines de puissance unitaire comprise généralement entre 250 et $350 \mathrm{MW}$ avec parfois des hauteurs de chutes très fortes, jusqu'à $800 \mathrm{~m}$.

Robinets de garde

Les robinets de garde de turbines ont également atteint des dimensions de plus en plus grandes. Neyrpic a été partie prenante à deux réalisations qui constituent des records mondiaux :

- les robinets-papillons de Tarbela $(\varnothing 7530 \mathrm{~mm})$;

— les robinets sphériques d'Alto Lindoso, en association avec Sorefame $(\varnothing 3400 \mathrm{~mm})$.

Pour permettre de conserver aux grandes turbines hydrauliques les qualités traditionnelles des turbomachines (excellent facteur d'utilisation, rapidité de mise en service et de prise en charge, souplesse d'utilisation), les constructeurs ont développé un important volume de recherches au niveau des conceptions hydraulique et mécanique ainsi que de la fabrication. 
Plusieurs articles de La Houille Blanche, ainsi que les $X X^{\text {es }}$ journées de l'Hydraulique ayant traité ces sujets, je me contenterai de rappeler quelques axes de recherches:

- l'élévation de niveau des performances hydrauliques;

— la prévision du comportement de la machine industrielle grâce aux études préalables sur modèle réduit en similitude ;

- la détermination beaucoup plus précise des contraintes et déformations statiques et dynamiques des différentes structures de machines;

- l'évolution des procédés de fabrication pour permettre de réaliser en toute sécurité des ensembles d'encombrement et de masse importants.

La course au gigantisme des années soixante-dix et quatre-vingts s'est traduite par une excellente maîtrise des performances hydrauliques et des conceptions mécaniques associées, ce qui a permis d'améliorer les performances hydrauliques tout en limitant les augmentations dimensionnelles.

L'analyse des projets ayant le plus de chance de se concrétiser dans les prochaines années montre que la course au gigantisme n'est plus autant d'actualité, sauf peut-être en Chine.

Ce n'est pas pour cela que les constructeurs vont pouvoir ralentir leurs efforts de Recherche et Développement. L'obtention de caractéristiques hydrauliques toujours améliorées reste une priorité, car une différence d'un dixième de pour cent de rendement ou une différence d'enfoncement de quelques dizaines de centimètres peuvent représenter des enjeux très importants pour le maître d'ouvrage.

D'autre part, l'équipement des faibles chutes, la mise au point de distributeurs ayant une tendance hydraulique à fermer sur toute leur course, l'amélioration des tracés pour les turbines-pompes mono étage de très haute chute, etc..., sont des domaines de développement tout à fait actuels.

Par ailleurs, le marché de la réhabilitation des centrales anciennes est en forte augmentation. Or il pose de difficiles problèmes aux constructeurs : augmentation des puissances à calage donné, nécessité de mélanger des composants très anciens (bâches, aspirateurs) avec des parties neuves (roues).

Les difficultés techniques à résoudre au cours des prochaines années ne seront donc plus tout à fait les mêmes que lors de la réalisation des Francis d'Itaipu, des Kaplan de Gezhouba ou des bulbes de Rock Island. Sauf exception les aspects dimensionnels perdront de leur acuité alors que les aspects hydrauliques resteront essentiels. Simultanément les outils de conception et d'analyse nécessiteront, grâce au développement d'outils de calculs tridimensionnels, un recours accru aux calculs par ordinateur en complément des essais sur modèles réduits. Ces derniers resteront indispensables pour valider en phase finale les résultats sortis des ordinateurs.

De même, le recours à des matériaux nouveaux (céramiques, matériaux amorphes, matériaux fabriqués à partir de composés organiques, etc...) devrait devenir de plus en plus fréquent.

Mais les constructeurs ne sont pas seulement confrontés à des challenges techniques. D'autres préoccupations les amènent à prendre des risques importants. Autrefois, le constructeur réalisait dans ses ateliers la quasitotalité des composants des turbines qu'il avait étudiées et vendues. Ce cas de figure est aujourd'hui l'exception. Le constructeur doit bâtir des schémas industriels complexes avec des partenaires ou des sous-traitants situés à l'étranger qui fabriquent une bonne partie des turbines, ce qui entraîne parfois des risques aussi préoccupants que les risques techniques ou technologiques.

François de VITRY 